\title{
Two small molecule compounds, LLL12 and FLLL32, exhibit potent inhibitory activity on STAT3 in human rhabdomyosarcoma cells
}

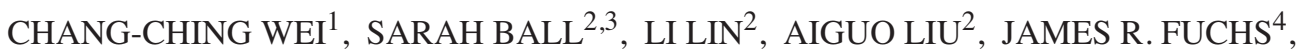 \\ PUI-KAI LI ${ }^{4}, \mathrm{CHENGLONG} \mathrm{LI}^{4}$ and JIAYUH LIN ${ }^{2,3}$ \\ ${ }^{1}$ Department of Pediatrics, China Medical University Hospital, Taichung, Taiwan, R.O.C.; ${ }^{2}$ Center for Childhood Cancer, \\ The Research Institute at Nationwide Children's Hospital, Department of Pediatrics, College of Medicine, \\ ${ }^{3}$ Molecular, Cellular, and Developmental Biology Program, ${ }^{4}$ Division of Medicinal Chemistry \\ and Pharmacognosy, College of Pharmacy, The Ohio State University, Columbus, OH, USA
}

Received August 10, 2010; Accepted October 22, 2010

DOI: 10.3892/ijo_00000848

\begin{abstract}
Signal transducers and activators of transcription 3 (STAT3) signaling is persistently activated in many types of cancer cells, and represents a valid target for anticancer drug design. However, few reports have described the constitutive activation of STAT3 in human sarcoma cells. In this study, we demonstrate that the STAT3 signaling pathway is constitutively activated in human rhabodomyosarcoma cells (RH28, RH30, and RD2). We also investigated the inhibitory effects of two newly developed small molecules, LLL12 and FLLL32, on the STAT3 signaling pathway in human rhabodomyosarcoma cells. Both LLL12 and FLLL32 downregulated STAT3 constitutively and interleukin-6 (IL-6) stimulated phosphorylated STAT3 (p-STAT3). The inhibition of STAT3 via LLL12 and FLLL32 was confirmed by the inhibition of STAT3 DNA binding activity. The downstream targets of STAT3, cyclin D1, Bcl-xL, and survivin were also down-
\end{abstract}

Correspondence to: Dr Jiayuh Lin, Center for Childhood Cancer, The Research Institute at Nationwide Children's Hospital, Department of Pediatrics, College of Medicine, The Ohio State University, 700 Children's Drive, Columbus, OH 43205, USA

E-mail:1in.674@osu.edu

Dr Chang-Ching Wei, Department of Pediatrics, China Medical University Hospital, No. 2 Yuh- Der Road, Taichung, 404 Taiwan, R.O.C.

E-mail: longus@seed.net.tw

Abbreviations: STAT3, signal transducers and activators of transcription 3; JAK2, Janus kinase 2; IL-6, interleukin-6; DMEM, Dulbecco's modified Eagle's medium; PARP, Poly(ADP-ribose) polymerase; pY705, phosphoryl tyrosine 705

Key words: signal transducers and activators of transcription 3 , IL-6, rhabdomyosarcoma regulated by LLL12 and FLLL 32 at both messenger RNA and protein levels. The potency of LLL12 and FLLL32 to inhibit proliferation/viability in human rhabodomyosarcoma cells (RH28, RH30, and RD2) was higher than that of the 5 previously reported Janus kinase 2 (JAK2)/ STAT3 inhibitors (LLL3, WP1066, Stattic, S3I-201, and AG490) and curcumin. Thus, in this study, we investigated the inhibitory effects of two STAT3 inhibitors, LLL12 and FLLL32, on the STAT3 signaling pathway in human rhabodomyosarcoma cells; we also demonstrated their higher potency in inhibiting proliferation on human rhabodomyosarcoma cells as compared to other five JAK2/STAT3 inhibitors and curcumin.

\section{Introduction}

Rhabdomyosarcoma, a highly malignant mesenchymal tumor, originating from immature striaed muscle, is the most common soft tissue sarcoma in children (1). Patients with rhabodomyosarcoma have a high relapse rate of $30 \%(1,2)$. Despite aggressive therapy, incorporating surgery, radiation therapy and dose-intensive chemotherapy, mortality rate of relapsing rhabodomyosarcoma is still $50-80 \%(2,3)$. Thus, development of higher potency and less toxicity anticancer drugs is pivotal to improve survival for patients with rhabdomyosarcoma.

Signal transducers and activators of transcription 3 (STAT3) signaling is constitutively activated in various types of cancer cells, such as breast cancer, prostate cancer, leukemia and lymphomas (4). Activated STAT3 binds the promoters of the targeted genes to control cell growth and survival. Thus, inhibition of STAT3 signaling pathway seems to be an attractive strategy for anticancer drug design $(5,6)$. For the past few years, several small molecules of the JAK2/STAT3 inhibitors, such as WP1066 (7-9), LLL3 (10) and AG490 (11-13), Stattic $(14,15)$ and S3I-201 $(16,17)$, have been reported with a wide range of antitumor effects. Although persistent activation of STAT3 has been detected in diverse cancer cell lines and tissue, only few reports have described elevated p-STAT3 in rhabdomyosarcoma. 
In this study, we present two newly developed small molecule STAT3 inhibitors, LLL12 and FLLL32, and analyzed their effects on reducing proliferation and inducing apoptosis, compared to the 5 previously reported JAK2/STAT3 inhibitors (LLL3, WP1066 and AG490, Stattic, and S3I-201) and curcumin in human rhabodomyosarcoma cells (RH28, RH30 and RD2). Both LLL12 and FLLL32 displayed high potency in inhibiting proliferation/viability over the other 6 compounds on human rhabodomyosarcoma cells.

\section{Materials and methods}

Antibodies and reagents. STAT3 inhibitors, LLL3, LLL12 and FLLL32, were synthesized in the Laboratory of Dr PuiKai Li (College of Pharmacy, The Ohio State University). The powder was dissolved in sterile dimethyl sulfoxide (DMSO) to make a $20 \mathrm{mmol} / \mathrm{l}$ stock solution. Aliquots of the stock solution were stored at $-20^{\circ} \mathrm{C}$. STAT3 inhibitors (S3I-201 and Stattic), JAK2 inhibitors (AG490 and WP1066) and curcumin were purchased from Calbiochem.

Cell lines and culture. Human rhabodomyosarcoma cell lines (RD2) was purchased from American Type Culture Collection. Human rhabodomyosarcoma cell lines (RH28 and RH30) were gifts from Peter Houghton. All cell lines were cultured in Dulbecco's modified Eagle's medium (DMEM) containing $10 \%$ fetal bovine serum (FBS) and $100 \mathrm{U} / \mathrm{ml}$ penicillin/ streptomycin/amphotericin B (Fisher Scientific International) in a $5 \% \mathrm{CO}_{2}$ incubator at $37^{\circ} \mathrm{C}$.

MTT cell viability assay. Approximately 3,000 cells of RH28, RH30, and RD2 were seeded in 96-well plates (3,000 cells/ well) in triplicates with $100 \mu 1$ 10\% FBS-supplemented DMEM medium overnight. Each cell line was treated with target medications with a series of concentrations for $72 \mathrm{~h}$. Cell viability was analyzed by the 3-(4,5-dimethyl-2-thiazolyl)-2, 5-diphenyl-2H-tetrazolium bromide (MTT) (Sigma, St. Louis, MO, USA) assay in three replicates. At the end-point, $25 \mu 1$ of MTT was added to each well of the plate and incubated for $3.5 \mathrm{~h}$. Cells were treated with MTT $(1 \mathrm{mg} / \mathrm{ml})$ for 3-4 h and then $100 \mu \mathrm{l}$ of N,N-dimethylformamide (D4551, Sigma) solubilization solution was added to each well. Colorimetric quantification was determined by an EL808 Ultra Micro-plate Reader (Bio-Tek Instruments, Inc) after the addition of formazan dissolved in $25 \% \mathrm{~N}, \mathrm{~N}$-dimethylformamide and $10 \%$ SDS under light-proof conditions overnight. Microsoft excel was used to analyze the cell viability data. The viability of the untreated cells was arbitrarily set at $100 \%$ and compared with the viability of cells treated with LLL12, FLLL32, selected JAK2/STAT3 inhibitors (LLL3, WP1066 and AG490, Stattic, and S3I-201), and curcumin. Error bars represent one standard deviation. Half-maximal inhibitory concentrations $\left(\mathrm{IC}_{50}\right)$ were calculated using Sigma Plot 9.0 software (Systat Software Inc.) using the 4 parameter logistic function standard curve analysis for dose response.

Western blot analysis. Rhabodomyosarcoma cells (RH28, RH30, and RD2) were treated with LLL12 or FLLL32 at a concentration of $5 \mu \mathrm{M}$ or $10 \mu \mathrm{M}$ or a DMSO vehicle control for $24 \mathrm{~h}$. For Western blotting, $60 \mu \mathrm{g}$ of total cell lysate was subjected to SDS polyacrylamide gel electrophoresis (PAGE) and transferred to PVDF membrane. The membranes were blocked with 5\% non-fat dry milk in TBST for $30 \mathrm{~min}$ and then blotted overnight with the following antibodies purchased from Cell Signaling Technologies: phospho-STAT3Y705 antibody (\#9131); STAT3 antibody (\#9132); cleaved Poly(ADP-ribose) polymerase (PARP) antibody (\#9546); cleaved caspase-3 antibody (\#9661) and GAPDH antibody (\#2118). Membranes were analyzed with enhanced chemiluminescence Plus reagents (GE Healthcare) and scanned with a Storm PhosphorImager (Amersham Pharmacia Biotech Inc.).

IL-6 induction of STAT3 phosphorylation. Rhabdomyosarcoma (RH28) cells were seeded in $10-\mathrm{cm}$ plates and allowed to adhere overnight. The following night, the cells were serumstarved. The cells were then left untreated or were treated with LLL12 (5 and $10 \mu \mathrm{M})$, FLLL32 (10 and $20 \mu \mathrm{M})$, or DMSO. After $3 \mathrm{~h}$, the untreated and LLL12-treated cells were stimulated by IL-6 $(10 \mathrm{ng} / \mathrm{ml})$. The cells were harvested at $30 \mathrm{~min}$ and analyzed by Western blotting.

DNA binding activity. Rhabodomyosarcoma cells (RH30) at $60-80 \%$ confluence were treated with LLL12 $(2.5$ or $5 \mu \mathrm{M})$, $5 \mu \mathrm{M}$ FLLL32, or DMSO in the presence of $10 \%$ FBS for $24 \mathrm{~h}$. The nuclear extracts were analyzed for STAT3 DNA binding activity using STAT3 Transcription Factor Kits following the manufacturer's protocol (Clontech, Inc.).

RT-PCR analysis. RNA was collected from human rhabdomyosarcoma cells (RH28, RH30, and RD2) with RNeasy Kits (Qiagen) following $24 \mathrm{~h}$ of treatment with LLL12 and FLLL32 at concentration of $10 \mu \mathrm{M}$. cDNA was generated from $500 \mathrm{ng}$ sample RNA using Omniscript RT (Qiagen). Two $\mu 1$ of cDNA was subsequently used for PCR. Primer sequences were as follows: (forward/reverse sequences) Bcl-xL (5'-TTGGACAATGGACTGGTTGA/GTAGAGTGGATGG TCAGTG-3'); Cyclin D1 (5'-GCTGGAGCCCGTGAAAA AGA/CTCCGCCTCTGGCATTTTG-3'); Survivin (5'ACCAGGTGAGAAGTGAGGGA/AACAGTAGAGGAGC CAGGGA-3'); GAPDH (5'-TGATGACATCAAGAAGGTG GTGAAG/TCCTTGGAGGCCATGTGGGCAT-3'). PCR amplifications were performed as follows: $5 \mathrm{~min}$ at $94^{\circ} \mathrm{C}$ followed by 25 cycles of $\left(30 \mathrm{sec}\right.$ at $94^{\circ} \mathrm{C}, 30 \mathrm{sec}$ at $55^{\circ} \mathrm{C}$, $30 \mathrm{sec}$ at $72^{\circ} \mathrm{C}$ ) and a final extension at $72^{\circ} \mathrm{C}$ for $5 \mathrm{~min}$. The PCR products were then run on $2 \%$ agarose gels, stained with ethidium bromide and visualized under UV light.

\section{Results}

LLL12, a STAT3 inhibitor. Phosphoryl tyrosine 705 (pY705) is crucial for the dimerization of STAT3 (18-20). Using a structure-based drug design, we developed a compound, LLL12 (Fig. 1A), which interferes with STAT3 dimerization by competitively binding to pY705. The phosphoryl tyrosine 705 is located on a loop segment of the SH2 domain and binds with several adjacent amino acid residues (leucine 706, threonine 708 , and phenylalanine 710 ) to a cavity on the SH2 domain of the other STAT3 monomer. To optimize potency and selectivity, the main scaffold of LLL12 contains fragments 
(A) LLL12<smiles>NS(=O)(=O)c1cccc2c1C(=O)c1cccc(O)c1C2=O</smiles>

(B) FLLL32

Figure 1. (A) The chemical structure of (A) LLL12 and (B) FLLL32.

(A) $\mathrm{RH} 30$

DMSO

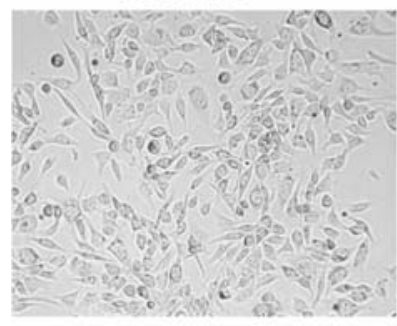

(B) RD2

DMSO

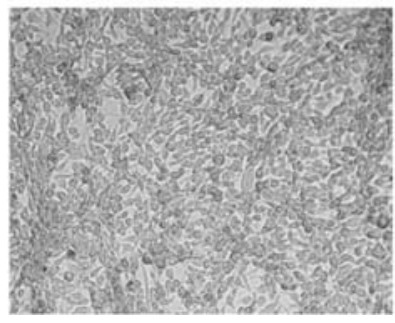

LLL12 $5 \mu \mathrm{M}$

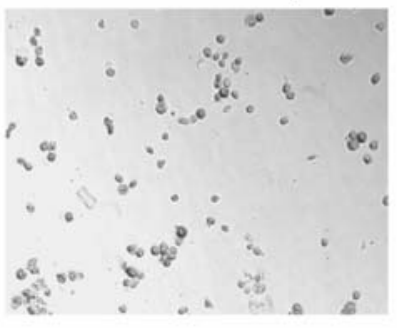

LLL12 $5 \mu \mathrm{M}$

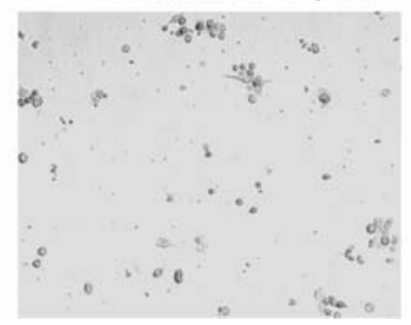

FLLL32 $5 \mu \mathrm{M}$

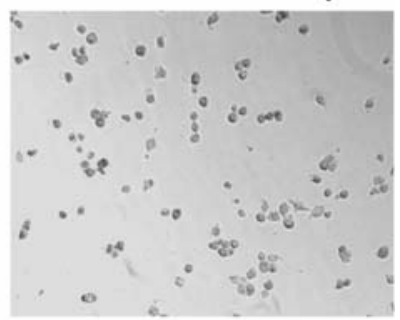

FLLL32 $5 \mu \mathrm{M}$

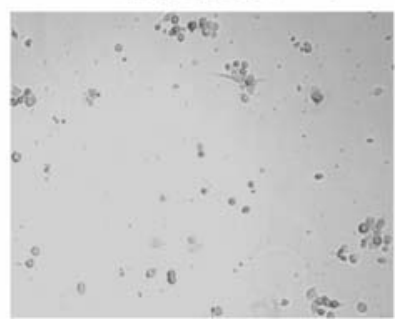

Figure 2. Inhibition of cell proliferation on human rhabdomyosarcoma cells (RH30 and RD2) was observed under phase contrast microscope (x100) after treatment with LLL12 $5 \mu \mathrm{M}$ or FLLL32 $5 \mu \mathrm{M}$ for $24 \mathrm{~h}$.

that directly contact the pY705 binding site of STAT3. A simulated docking model shows that the carboxylic and sulfonamide tails of LLL12 occupy the pY705 binding pocket of STAT3. The simulated binding energies of LLL12 to STAT3 predict it to be a potent inhibitor of the constitutive STAT3 pathway. The detail of LLL12 synthesis was described in our previous report (21).

FLLL32, a JAK2/STAT3 inhibitor. FLLL32 is a diketone analogue of curcumin (Fig. 1B). The central B-dicarbonyl moiety of curcumin is subjected to keto-enol tautomerization, which is hypothesized to influence its target selectivity by virtue of its biological activity. Replacing the 2 hydrogen atoms on the central carbon of curcumin with a spiro-cyclohexyl ring in FLLL32, eliminated the ability of curcumin to enolize. These FLLL32 modifications are predicted to make it interact better with key binding sites of JAK2 and the $\mathrm{SH} 2$ domain of STAT3 than the keto-enol form of curcumin (22). In addition, FLLL32 is characterized as a 3,4-dimethoxy substituent that mimics dimethoxycurcumin, which has shown increased stability, higher plasma concentration, and greater efficacy against cancer cells than standard curcumin (22).

Inhibition of cell viability by LLL12 and FLLL32 in human rhabdomyosarcoma cells. STAT3 activation is important for tumor cell proliferation and survival $(19,23)$. We observed the total cell number reduction of human rhabodomyosarcoma cells (RH30 and RD2) after treatment with LLL12 or FLLL32 at a concentration of $5 \mu \mathrm{M}$ for $24 \mathrm{~h}$ compared to those treated with DMSO vehicle under phase contrast microscope (Fig. 2). We further examined the inhibitory effect of LLL12 and FLLL32 on human rhabodomyosarcoma cells (RH28, RH30, and RD2) through the MTT viability assay. A dose-dependent inhibition in tumor cell proliferation/viability was seen after 72-h treatment (Fig. 3).

LLL12 and FLLL32 inhibited STAT3 phosphorylation and induced apoptosis in human rhabodomyosarcoma cells. Firstly, we detected constitutively activated STAT3 in human 
(A) LLL12

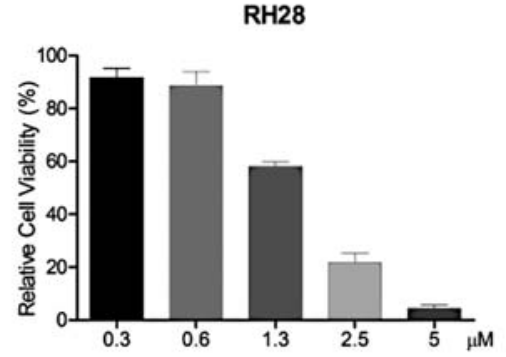

(B) FLLL32

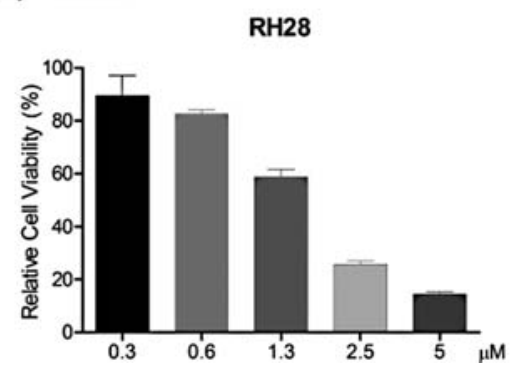

RH30

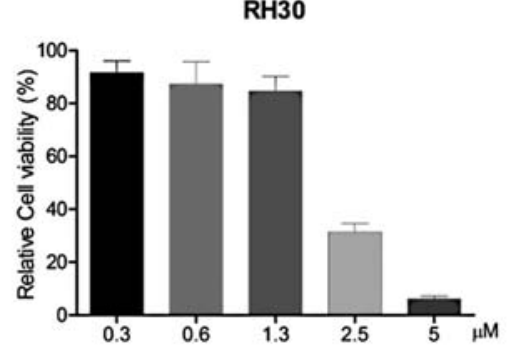

RH30

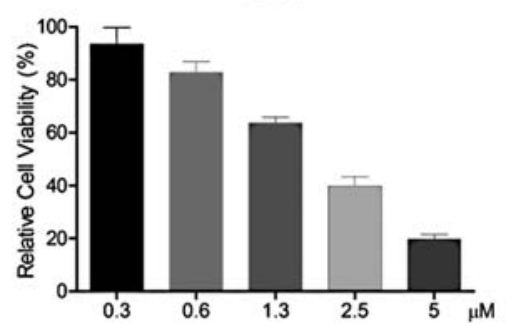

RD2

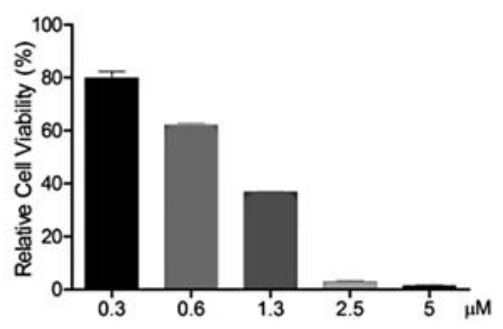

RD2

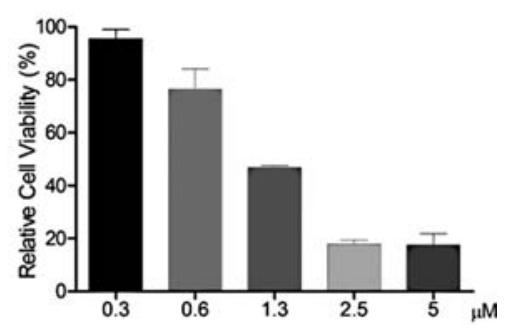

Figure 3. (A) LLL12 and (B) FLLL32 inhibited proliferation/viability of human rhabdomyosarcoma cells (RH28, RH30 and RD2), as determined by the MTT assay. A dose-dependent inhibition in tumor cell proliferation/viability was observed after 72-h treatment.
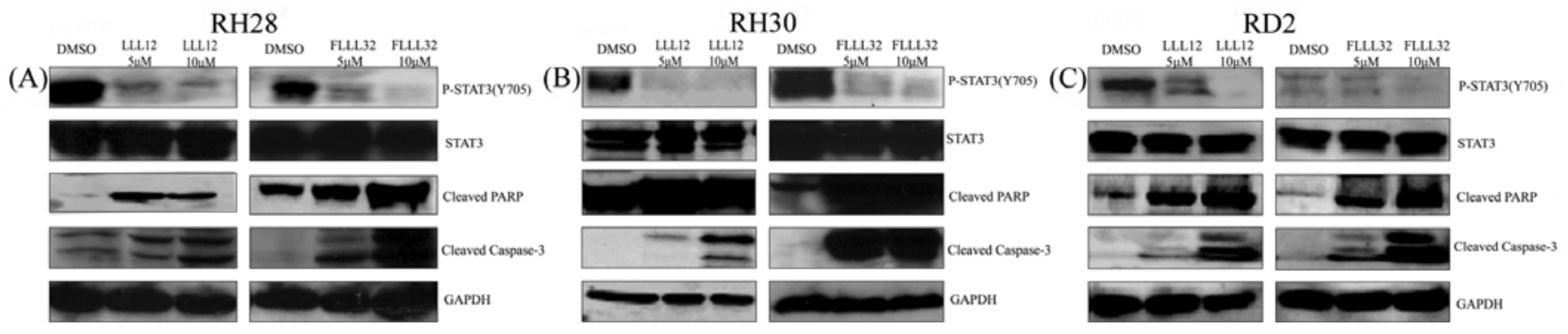

Figure 4. Western blot analysis showed that LLL12 and FLLL32 inhibited STAT3 phosphorylation on human rhabdomyosarcoma cells (RH28, RH30, and RD2) and induced apoptosis through the induction of cleaved caspase-3 and cleaved PARP.

rhabodomyosarcoma cells (RH28, RH30, and RD2) by Western blotting with GAPDH as an internal control and IFN- $\gamma$ stimulated HeLa cells as a positive control (data not shown). Then, RH28, RH30, and RD2 cells were separated into the first 3 groups treated with DMSO, $5 \mu \mathrm{M}$ LLL12 and $10 \mu \mathrm{M}$ LLL12 and into further 3 groups treated with DMSO, $5 \mu \mathrm{M}$ FLLL32 and $10 \mu \mathrm{M}$ FLLL32. Protein samples were collected for Western blot analysis after treatment for $24 \mathrm{~h}$. Both LLL12 and FLLL32 inhibited STAT3 phosphorylation at tyrosine residue 705 (Tyr 705) in the 3 cells lines. Upregulation of cleaved PARP and cleaved caspase-3 suggested that inhibition of STAT3 phosphorylation induced apoptosis (Fig. 4). Combined with cell viability assay data, LLL12 and FLLL32 not only suppressed cell growth/viability of rhabodomyosarcoma cells expressing constitutively active STAT3 but also induced cell apoptosis resulting a reduction of total cell number.

LLL12 and FLLL32 inhibited STAT3 phosphorylation induced by $I L-6$. STAT3 activation can be induced by IL-6 (24-26). Human rhabdomyosarcoma (RH28) cells were used to determine if LLL12 and FLLL32 can inhibit IL-6-induced
STAT3 phosphorylation. We found that the basal levels of STAT3 phosphorylation are high even at serum-free medium for 24 h. IL-6 slightly increased STAT3 phosphorylation in RH28 cells and the increasing was blocked by LLL12, and was blocked to a lesser degree by FLLL32 because FLLL32 is diketone analogues of curcumin, which is less soluble in serum-free medium (22). This inhibitory effect was dosedependent (Fig. 5).

LLL12 and FLLL32 inhibited STAT3 DNA binding. To confirm the inhibition of STAT3 signaling by LLL12 and FLLL32, we examined the inhibition of STAT3 DNA binding activity. Both LLL12 and FLLL32 caused a statistically significant inhibition of STAT3 DNA binding activity in human rhabodomyosarcoma cells (RH30) (Fig. 6).

LLL12 and FLLL32 inhibited the transcription of downstream targets of STAT3 on human rhabdomyosarcoma cells. To further analyze the mechanism of inhibition of STAT3 by LLL12 and FLLL32 on the inhibition of STAT3, we examined the transcription of downstream target genes of STAT3, such as cyclin D1, survivin, and Bcl-xL, by reverse transcriptase 


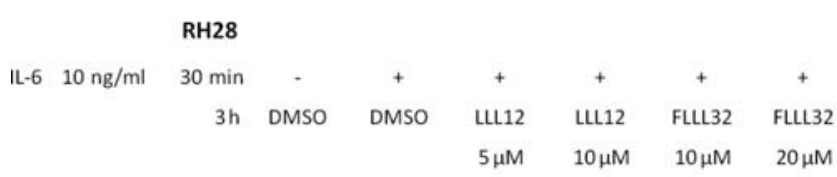

p-STAT3(Y705)

STAT3

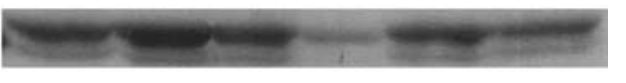

GAPDH

Figure 5. Western blot analysis demonstrated that LLL12 and FLLL32 inhibited STAT3 phosphorylation induced by IL-6 (serum-starved medium) in human rhabdomyosarcoma cells (RH28). Since FLLL32 was less soluble in serum-starved medium, it revealed less inhibitory effect on STAT3 phosphorylation compared to LLL12 in RH28.

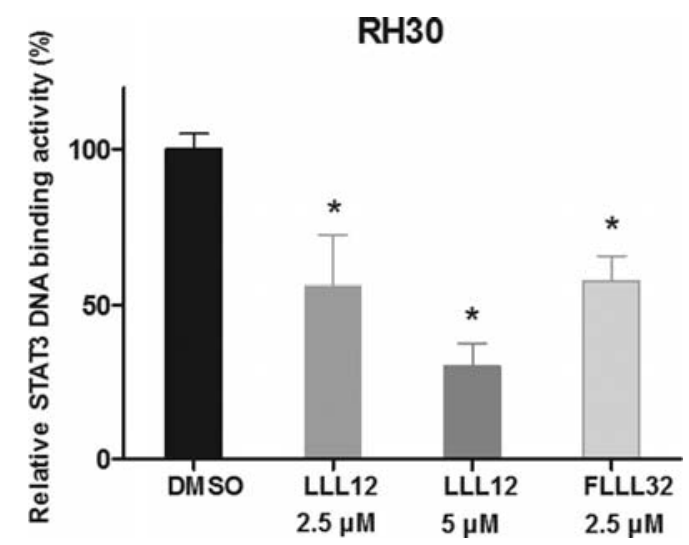

Figure 6. LLL12 and FLLL32 have inhibitory effects on STAT3 DNA binding activity and STAT3-dependent transcriptional activity. The nuclear extracts of RH30 cancer cells were analyzed for STAT3 DNA binding. Statistical significance $(\mathrm{P}<0.05)$ relative to the DMSO vehicle control is designated by an asterisk.

PCR (RT-PCR). STAT3 is known to upregulate cyclin D1, which promotes cell cycle progression and growth factor independence, and to upregulate Bcl-xL and survivin, which inhibit apoptosis. We treated human rhabodomyosarcoma cells (RH28, RH30, and RD2) with FLLL32 (10 $\mu \mathrm{M})$, LLL12 $(10 \mu \mathrm{M})$, or DMSO for $24 \mathrm{~h}$. We found that LLL12 or FLLL32 resulted in downregulation of transcription of STAT3-regulated genes, cyclin D1, survivin, and Bcl-xL in 3 cell lines (Fig. 7).

LLL12 and FLLL32 showed higher potency in inhibiting proliferation/viability compared to selected JAK2/STAT3 inhibitors and curcumin in human rhabdomyosarcoma cells. We analyzed the inhibitory effects of LLL12 and FLLL32 on proliferation/viability and compared their inhibitory effects with other previously reported JAK2/STAT3 inhibitors (LLL3, WP1066, Stattic, S3I-201, and AG490) and curcumin in human rhabodomyosarcoma cells (RH28, RH30 and RD2). A dose-dependent inhibition in cell proliferation/viability was observed after 72-h of treatment. Based on $\mathrm{IC}_{50}$ values calculated for LLL12, FLLL32, and other JAK2/STAT3 inhibitors (Table I), both LLL12 and FLLL32 showed superior growth suppressive activity compared to other JAK2/STAT3 inhibitors (LLL3, WP1066, Stattic, S3I-201, and AG490) and curcumin on RH28, RH30, and RD2.

\section{Discussion}

To our knowledge, this is the first study to demonstrate the inhibitory effects of 2 newly developed small molecule compounds, LLL12 and FLLL32, on STAT3 signaling pathway in human rhabodomyosarcoma cells (RH28, RH30 and RD2). Both LLL12 and FLLL32 presented more potent antiproliferative effects than the 5 previously reported drugs targeting the JAK2/STAT3 signaling pathway (LLL3, WP1066, Stattic, S3I-201, and AG490) and curcumin in human rhabodomyosarcoma cells. Our study is one of the few showing the constitutive activation of STAT3 in human rhabdomyosarcoma cell lines as well as demonstrating the effects of reducing proliferation and inducing apoptosis by treating these cells with STAT3 inhibitors.

Recent studies have successfully demonstrated the role of aberrant STAT3 in malignant transformation and tumorigenesis $(19,23,27)$. Activation of the upstream cytokine and growth factor receptors induces STAT3 phosphorylation at tyrosine residue 705, dimerization through reciprocal $\mathrm{SH} 2$ domains, and translocation into the nucleus. Then, phosphorylated STAT3 binds target genes to control cell growth and survival (28). Growing evidence from both cellular and animal systems
(A) $\mathrm{RH} 28$

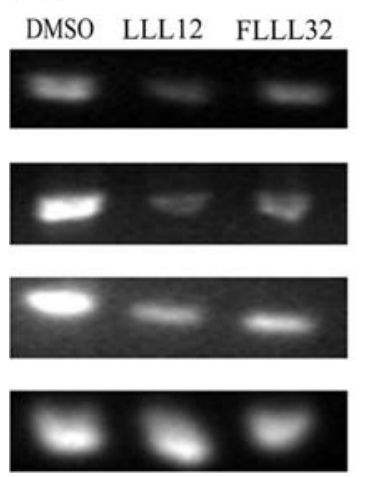

(B) $\mathrm{RH} 30$
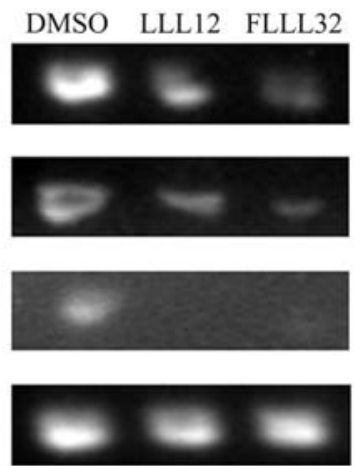

(C) RD2
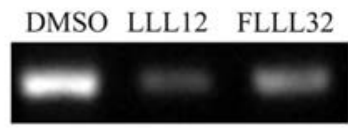

Survivin
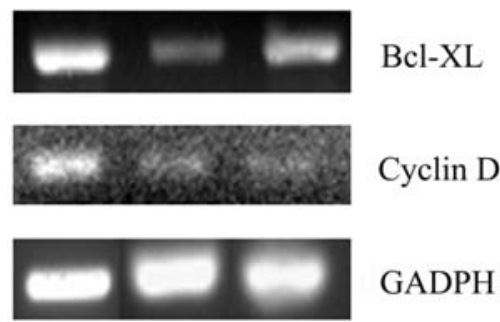

Cyclin D1

GADPH

Figure 7. RT-PCR revealed that LLL12 and FLLL32 inhibited the transcription of STAT3 downstream target genes associated with cell growth and survival (cyclin D1, survivin, and Bcl-xL) in human rhabdomyosarcoma cells (RH28, RH30, and RD2). 
Table I. Half-maximal inhibitory concentrations $\left(\mathrm{IC}_{50}\right)(\mu \mathrm{M})$ obtained for LLL12, FLLL32, JAK2/STAT3 inhibitors (LLL3, WP1066, Stattic, S3I-201, and AG490), and curcumin in human rhabdomyosarcoma cells (RH28, RH30, and RD2).

\begin{tabular}{lcccccccc}
\hline & LLL3 & LLL12 & FLLL32 & WP1066 & Stattic & S3I-201 & AG490 & Curcumin \\
\hline RH28 & 22.32 & 1.74 & 1.40 & 9.20 & 2.31 & 55.95 & $>60$ & 17.32 \\
RH30 & 33.20 & 2.29 & 1.83 & 5.09 & 2.55 & $>60$ & $>60$ & 27.82 \\
RD2 & 17.05 & 0.98 & 0.94 & 4.97 & 1.48 & $>60$ & $>60$ & 16.37 \\
\hline
\end{tabular}

All values reflect concentrations calculated after 72-h treatment in a MTT viability assay.

has shown that the inhibition of constitutively active STAT3 induces apoptosis in cancer cells (29-32). STAT3 has such crucial roles in human cancer formation as to be a valid target for novel anticancer drug design $(28,33)$. Although various compounds have been reported to inhibit the STAT3 signaling pathway, such as DNA decoys, dominant-negative STAT3 mutants, STAT3 small interfering RNAs (siRNAs), or antisense STAT3 oligonucleotides, the development of smaller and more effective compounds by either manipulating the potency or improving the pharmacokinetics by reducing the molecule weight is a feasible strategy in drug design (34). In addition, targeting central protein directly to avoid adverse effects is also important in developing new anticancer agents. Several target sites have been reported in designing STAT3 inhibitors. Selective inhibition of JAK2 can prevent STAT3 phosphorylation and dimerization. Several JAK2 inhibitors, such as AG490, WP1066, and SD-1029 have been reported in recent studies (7-13). Selective competition of SH2 domain which is crucial for STAT3 dimerization can disrupt STAT3 signaling. Several non-peptide small molecule $\mathrm{SH} 2$ inhibitors, such as Stattic and S3I-201, have also been recently reported $(14,16)$.

We have been developing a series of compounds that competitively bind to pY705 of STAT3 and then interfere with STAT3 dimerization and activation. Interestingly, LLL12 has been the most potent inhibitor of the STAT3 signaling pathway among these compounds. Our previous findings have shown the inhibitory effects on STAT3 activation and cell proliferation of some of these series compounds on different cancer cells, such as STA-21 on human breast, prostate, and bladder cancers (35-37), and LLL3 on human prostate cancer and glioblastoma $(10,36)$. The main scaffold of LLL3 only binds to the side pocket in close proximity to the pY705 binding site of STAT3 monomer but does not bind directly to the dimerization site, pY705. To optimize the potency and selectivity, we further design a compound attached to the main scaffold that contact pY705 (LLL12; Fig. 1A). The simulated docking mode of LLL12 is similar to LLL3 but with the carboxylic and sulfonamide tails occupying the pY705 binding pocket of STAT3. For simulated binding energies to STAT3, LLL12 is 10 -fold better than LLL3 and is predicted to be more potent than LLL3 in inhibiting constitutive STAT3 pathway. Recently, we reported the potent inhibition of STAT3 phosphorylation and growthsuppression of LLL12 in human breast cancer and glioblastoma cells (21).

A newly developed small molecule STAT3 inhibitor, FLLL32, has recently been developed from curcumin in our laboratory. Curcumin (diferuloylmethane) is a leading compound of perennial herb Curcuma longa (22). Almost 3,000 studies carried out with curcumin suggest that this natural agent has a diverse range of molecular targets, including transcription factors, growth factors, cytokines, enzymes, and genes regulating cell proliferation and apoptosis (38-45). The complex effects of curcumin allow it to inhibit multiple oncogenic processes, including those associated with the JAK2/STAT3 pathway. Although curcumin is pharmacologically safe in humans based on nearly 40 clinical trials, its low bioavailability is a concern. FLLL32 shares many of the bioactive properties of curcumin based on their similar structures. Designed to preferentially interact with both the SH2 domain of STAT3 and its upstream activator JAK2, FLLL32 has greater stability and higher selectivity to critical domains in JAK2 and STAT3 compared to curcumin. We also demonstrated the potent inhibition of STAT3 phosphorylation and growth-suppression of FLLL32 in human breast and pancreatic cancer cells (21).

In this study, constitutive activation of STAT3 was observed in human rhabodomyosarcoma cells, and inhibition of STAT3 activity reduced proliferation and induced apoptosis on these cells. Although STAT3 is found to be constitutively activated in human tumors, reports on activated STAT3 in sarcoma are rare. One study showed nearly 50\% Ewing sarcoma tissue presenting STAT3 activation (46). We recently analyzed sarcoma tissue microarray slides and sarcoma cell lines and found elevation of activated STAT3 detected in approximately $25 \%$ sarcoma cells, including human rhabodomyosarcoma, osteosarcoma, and other soft tissue sarcomas (47). We also treated the human rhabodomyosarcoma cells with a low molecular weight compound STA-21 or block STAT3 signaling by dominant-negative STAT3 mutant, both of which induced rhabdomyosarcoma cell growth inhibition and apoptosis $(37,47)$. Persistently activated STAT3 was also found in canine osteosarcoma (48). Our current study extended previous findings to show that blocking constitutive STAT3 activation in rhabodomyosarcoma cells inhibited cell growth and induced apoptosis through newly developed small molecule inhibitors and other JAK2/STAT3 inhibitors. Furthermore, LLL12 and FLLL32 inhibited the expression of the STAT3-regulated genes encoding cyclin D1, Bcl-xL, and survivin and inhibited the growth of human rhabodomyosarcoma cells in vivo (5).

In conclusion, the 2 small-molecular STAT3 inhibitors, LLL12 and FLLL32, have higher potency in inhibiting tumor growth compared to the 5 previously reported JAK2/STAT3 
inhibitors and curcumin on rhabodomyosarcoma cells (RH28, RH30 and RD2). Further in vivo mouse tumor model, pharmacodynamic, and pharmacokinetic studies should be conducted to explore the future clinical potential of STAT3 inhibitors in rhabodomyosarcoma cells harboring aberrant STAT3 signaling.

\section{Acknowledgements}

This study was funded by China Medical University Hospital (DMR-94-023).

\section{References}

1. Koscielniak E, Morgan M and Treuner J: Soft tissue sarcoma in children: prognosis and management. Paediatr Drugs 4: 21-28, 2002.

2. Crist W, Gehan EA, Ragab AH, et al: The third intergroup rhabdomyosarcoma study. J Clin Oncol 13: 610-630, 1995.

3. Klingebiel T, Pertl U, Hess CF, et al: Treatment of children with relapsed soft tissue sarcoma: report of the german CESS/CWS REZ 91 trial. Med Pediatr Oncol 30: 269-275, 1998.

4. Bromberg J and Darnell JE Jr: The role of STATs in transcriptional control and their impact on cellular function. Oncogene 19: 2468-2473, 2000.

5. Buettner R, Mora LB and Jove R: Activated STAT signaling in human tumors provides novel molecular targets for therapeutic intervention. Clin Cancer Res 8: 945-954, 2002.

6. Huang S: Regulation of metastases by signal transducer and activator of transcription 3 signaling pathway: clinical implications. Clin Cancer Res 13: 1362-1366, 2007.

7. Ferrajoli A, Faderl S, Van Q, et al: WP1066 disrupts janus kinase-2 and induces caspase-dependent apoptosis in acute myelogenous leukemia cells. Cancer Res 67: 11291-11299, 2007.

8. Iwamaru A, Szymanski S, Iwado E, et al: A novel inhibitor of the STAT3 pathway induces apoptosis in malignant glioma cells both in vitro and in vivo. Oncogene 26: 2435-2444, 2007

9. Verstovsek S, Manshi T, Quintas-Cardama A, et al: WP1066, a novel JAK2 inhibitor, suppresses proliferation and induces apoptosis in erythroid human cells carrying the JAK2 V617F mutation. Clin Cancer Res 14: 788-796, 2008.

10. Fuh B, Sobo M, Cen L, et al: LLL-3 inhibits STAT3 activity, suppresses glioblastoma cell growth and prolongs survival in a mouse glioblastoma model. Br J Cancer 100: 106-112, 2009.

11. Caceres-Cortes JR: A potent anti-carcinoma and anti-acute myeloblastic leukemia agent, AG490. Anticancer Agents Med Chem 8: 717-722, 2008.

12. Chen HF, Deng HY and Fan WK: Effect of janus kinase inhibitor AG490 on invasion and metastasis of human breast cancer cells. Sichuan Da Xue Xue Bao Yi Xue Ban 39: 63-65, $83,2008$.

13. Huang C, Cao J, Huang KJ, et al: Inhibition of STAT3 activity with AG490 decreases the invasion of human pancreatic cancer cells in vitro. Cancer Sci 97: 1417-1423, 2006.

14. McMurray JS: A new small-molecule Stat3 inhibitor. Chem Biol 13: 1123-1124, 2006.

15. Schust J, Sperl B, Hollis A, Mayer TU and Berg T: Stattic: a small-molecule inhibitor of STAT3 activation and dimerization. Chem Biol 13: 1235-1242, 2006.

16. Siddiquee K, Zhang S, Guida WC, et al: Selective chemical probe inhibitor of Stat3, identified through structure-based virtual screening, induces antitumor activity. Proc Natl Acad Sci USA 104: 7391-7396, 2007

17. Siddiquee KA, Gunning PT, Glenn M, et al: An oxazole-based small-molecule Stat3 inhibitor modulates Stat3 stability and processing and induces antitumor cell effects. ACS Chem Biol 2: 787-798, 2007.

18. Turkson J, Bowman T, Garcia R, Caldenhoven E, De Groot RP and Jove R: Stat 3 activation by Src induces specific gene regulation and is required for cell transformation. Mol Cell Biol 18: 2545-2552, 1998 .

19. Bowman T, Garcia R, Turkson J and Jove R: STATs in oncogenesis. Oncogene 19: 2474-2488, 2000.

20. Bromberg JF: Activation of STAT proteins and growth control. Bioessays 23: 161-169, 2001.
21. Lin L, Hutzen B, Li P, et al: A novel small molecule, LLL12, inhibits STAT3 phosphorylation and activities and exhibits potent growth-suppressive activity in human cancer cell. Neoplasia 12: 39-50, 2010.

22. Lin L, Hutzen B, Zuo M, et al: Novel STAT3 phosphorylation inhibitors exhibit potent growth-suppressive activity in pancreatic and breast cancer cells. Cancer Res 70: 2445-2454, 2010.

23. Frank DA: STAT3 as a central mediator of neoplastic cellular transformation. Cancer Lett 251: 199-210, 2007.

24. Shouda T, Hiraoka K, Komiya S, et al: Suppression of IL-6 production and proliferation by blocking STAT3 activation in malignant soft tissue tumor cells. Cancer Lett 231: 176-184, 2006.

25. Hodge DR, Hurt EM and Farrar WL: The role of IL-6 and STAT3 in inflammation and cancer. Eur J Cancer 41: 2502-2512, 2005.

26. Atreya R and Neurath MF: Signaling molecules: the pathogenic role of the IL-6/STAT-3 trans signaling pathway in intestinal inflammation and in colonic cancer. Curr Drug Targets 9: 369-374, 2008

27. Calo V, Migliavacca M, Bazan V, et al: STAT proteins: from normal control of cellular events to tumorigenesis. J Cell Physiol 197: 157-168, 2003.

28. Darnell JE: Validating Stat3 in cancer therapy. Nat Med 11: 595-596, 2005.

29. Leeman RJ, Lui VW and Grandis JR: STAT3 as a therapeutic target in head and neck cancer. Expert Opin Biol Ther 6: 231-241, 2006.

30. Battle TE and Frank DA: The role of STATs in apoptosis. Curr Mol Med 2: 381-392, 2002.

31. Smithgall TE, Briggs SD, Schreiner S, Lerner EC, Cheng H and Wilson MB: Control of myeloid differentiation and survival by stats. Oncogene 19: 2612-2618, 2000.

32. Jing $\mathrm{N}$ and Tweardy DJ: Targeting Stat3 in cancer therapy. Anticancer Drugs 16: 601-607, 2005.

33. Turkson J: STAT proteins as novel targets for cancer drug discovery. Expert Opin Ther Targets 8: 409-422, 2004.

34. Al Zaid Siddiquee K and Turkson J: STAT3 as a target for inducing apoptosis in solid and hematological tumors. Cell Res 18: 254-267, 2008

35. Chen CL, Cen L, Kohout J, et al: Signal transducer and activator of transcription 3 activation is associated with bladder cancer cell growth and survival. Mol Cancer 7: 78, 2008.

36. Bhasin D, Cisek K, Pandharkar T, et al: Design, synthesis, and studies of small molecule STAT3 inhibitors. Bioorg Med Chem Lett 18: 391-395, 2008.

37. Song H, Wang R, Wang S and Lin J: A low-molecular-weight compound discovered through virtual database screening inhibits Stat 3 function in breast cancer cells. Proc Natl Acad Sci USA 102: 4700-4705, 2005.

38. Karunagaran D, Rashmi R and Kumar TR: Induction of apoptosis by curcumin and its implications for cancer therapy. Curr Cancer Drug Targets 5: 117-129, 2005.

39. Araujo CC and Leon LL: Biological activities of curcuma longa L. Mem Inst Oswaldo Cruz 96: 723-728, 2001.

40. Shishodia S, Chaturvedi MM and Aggarwal BB: Role of curcumin in cancer therapy. Curr Probl Cancer 31: 243-305, 2007.

41. Bright JJ: Curcumin and autoimmune disease. Adv Exp Med Biol 595: 425-451, 2007.

42. Gautam SC, Gao X and Dulchavsky S: Immunomodulation by curcumin. Adv Exp Med Biol 595: 321-341, 2007.

43. Reuter S, Eifes S, Dicato M, Aggarwal BB and Diederich M: Modulation of anti-apoptotic and survival pathways by curcumin as a strategy to induce apoptosis in cancer cells. Biochem Pharmacol 76: 1340-1351, 2008.

44. Pari L, Tewas D and Eckel J: Role of curcumin in health and disease. Arch Physiol Biochem 114: 127-149, 2008.

45. Menon VP and Sudheer AR: Antioxidant and anti-inflammatory properties of curcumin. Adv Exp Med Biol 595: 105-125, 2007.

46. Lai R, Navid F, Rodriguez-Galindo C, et al: STAT3 is activated in a subset of the Ewing sarcoma family of tumours. J Pathol 208: 624-632, 2006.

47. Chen CL, Loy A, Cen L, et al: Signal transducer and activator of transcription 3 is involved in cell growth and survival of human rhabdomyosarcoma and osteosarcoma cells. BMC Cancer 7: $111,2007$.

48. Fossey SL, Liao AT, McCleese JK, et al: Characterization of STAT3 activation and expression in canine and human osteosarcoma. BMC Cancer 9: 81, 2009. 